\title{
Factors Associated With Treatment Adherence and Satisfaction in Type 2 Diabetes Management in Japan: Results From a Web-Based Questionnaire Survey
}

\author{
Ryo Suzuki · Shun Saita · Nobuhiro Nishigaki · Koichi Kisanuki • \\ Yukio Shimasaki · Tomoka Mineyama · Masato Odawara
}

Received: March 12, 2021 / Accepted: June 10, 2021 / Published online: July 20, 2021

(C) The Author(s) 2021

\begin{abstract}
Introduction: To identify factors associated with treatment adherence and satisfaction in patients with type 2 diabetes (T2DM) in Japan. Methods: A web-based questionnaire survey was conducted from 6 to 17 March 2019 in patients with $\mathrm{T} 2 \mathrm{DM}$ aged $\geq 20$ years receiving diabetes treatment. Treatment adherence and satisfaction were self-assessed/reported by the patients. A multiple logistic regression model and the chi-square test were used to assess associated factors.
\end{abstract}

Results: Responders $(N=1000)$ were aged 63.8 (standard deviation 11.9) years, and 739 (73.9\%) were male. Adherence to treatment was reported in 941 (94.1\%) patients and was significantly associated with higher household income (odds ratio [OR] 2.07, 95\% confidence

Supplementary Information The online version contains supplementary material available at https:// doi.org/10.1007/s13300-021-01100-3.

R. Suzuki · M. Odawara

Department of Diabetes Endocrinology and

Metabolism, Tokyo Medical University, Shinjuku-

$\mathrm{ku}$, Tokyo, Japan

S. Saita $(\varangle) \cdot$ N. Nishigaki · K. Kisanuki ·

Y. Shimasaki · T. Mineyama

Japan Medical Office, Japan Pharma Business Unit,

Takeda Pharmaceutical Company Limited, 2-1-1

Nihonbashi-Honcho, Chuo-ku, Tokyo 103-8668,

Japan

e-mail: Shun.saita@takeda.com interval [CI] 1.11-3.86), age (OR 1.04, 95\% CI 1.02-1.07), employment (OR 0.30, 95\% CI $0.15-0.60)$ and having $\geq 1$ impaired basic activity of daily living (BADL) (OR 0.33, 95\% CI 0.13-0.82). Satisfaction with treatment was reported by $575(57.5 \%)$ and was significantly associated with receiving/understanding guidance on how pharmacologic therapies are tailored (OR 1.73, 95\% CI 1.19-2.51), male sex (OR 1.55, 95\% CI 1.10-2.19), higher household income (OR 1.45, 95\% CI 1.09-1.94) and age (OR 1.02, 95\% CI 1.00-1.03). Treatment adherence was negatively associated with lower household income and having $\geq 1$ impaired BADL in patients aged $<65$ years, but not in those aged $\geq 65$ years. Treatment satisfaction was positively associated with higher household income and receiving/understanding guidance on exercise therapy and the importance of achieving target haemoglobin A1c levels in patients aged $\geq 65$ years, but with receiving/ understanding guidance on the tailoring of pharmacologic therapies in patients aged $<65$ years.

Conclusion: Lower age, lower household income, employment and impaired BADL may negatively impact treatment adherence in patients with T2DM. Appropriate physician guidance may promote treatment satisfaction. Differences in perspectives between patients aged $<65$ and those aged $\geq 65$ years should be considered. 
Trial Registration: Japan Pharmaceutical Information Center, JapicCTI-194636.

Keywords: Diabetes mellitus; Type 2; Medication adherence; Physician-patient relations; Surveys and questionnaires

\section{Key Summary Points}

Why carry out this study?

Treatment adherence and satisfaction impact the quality of diabetes care.

We investigated factors associated with treatment adherence and satisfaction in patients with type 2 diabetes (T2DM) in Japan.

\section{What was learned from this study?}

Lower age, lower household income, employment and impaired basic activity of daily living may negatively impact treatment adherence in patients with T2DM.

Receiving and understanding guidance on how pharmacologic therapies are tailored, higher household income, male sex and higher age may positively impact treatment satisfaction in patients with T2DM.

Different approaches may be effective when attempting to improve T2DM treatment adherence and satisfaction in patients aged $<65$ and $\geq 65$ years in Japan.

\section{INTRODUCTION}

Type 2 diabetes mellitus (T2DM) is caused by multiple genetic factors associated with reduced insulin secretion or sensitivity, accompanied by environmental factors such as overeating, lack of exercise, obesity, stress and ageing [1,2]. The standard treatment of T2DM recommended by the American Diabetes Association and the Japanese Diabetes Society (JDS) begins with medical nutrition/exercise therapy; if adequate glycaemic control is not achieved despite the continuation of these interventions, pharmacologic therapy is recommended [3, 4]. Patients with diabetes are responsible for self-management of their diet and lifestyle habits, monitoring blood glucose and taking medications in a timely fashion $[3,4]$.

Prolonged hyperglycaemia increases the risk of developing microvascular and macrovascular complications [4], but this risk can be reduced by achieving good blood glucose control [5-7]. To prevent microvascular complications, the JDS sets the target haemoglobin A1c (HbA1c) level in patients with diabetes at $<7 \%$ (or $8 \%$ when side effects or other factors prohibit treatment intensification) [4]. By avoiding diabetic complications, patients with T2DM can maintain a good quality of life (QoL) and have a life expectancy comparable to that of healthy individuals $[4,8]$. However, the proportion of patients in Japan achieving their target HbA1c level is suboptimal-approximately $50 \%$ of the patients under treatment $[5,9]$. Poor blood glucose control not only significantly impacts the life expectancy and QoL of patients with T2DM, but also healthcare costs [10].

Poor medication adherence is associated with poor blood glucose control [3, 11-13]; therefore, risk factors for poor adherence have been extensively studied so that prevention efforts can be implemented. Known risk factors for low treatment adherence include younger age [14-16], poor glycaemic control [17, 18] and high treatment cost [14-16]. Other factors have shown different results across studies and study populations; these include gender [14-17, 19], polypharmacy [14-18] and higher education $[14,15]$. In Japan, employment has been associated with poor treatment adherence [20].

Good medication adherence is not only associated with good blood glucose control, but also treatment satisfaction [21-23]. The importance of treatment satisfaction in diabetes management is well recognised [21, 23, 24]. A number of questionnaires assessing patient-reported outcomes including treatment satisfaction, wellbeing and QoL have been developed 
$[21,25]$ and used to identify sociodemographic and clinical factors associated with treatment satisfaction in many countries. Of these, common positive factors include male sex $[23,26]$, employment [27, 28], diabetes education $[28,29]$ and good glycaemic control $[22,26,29,30]$. Other factors have shown both positive and negative associations; these include age $[27,30]$ and presence of diabetic complications [23, 26, 27, 29, 31].

As daily self-management of diet, exercise and medication is the foundation of diabetes treatment, it may be effective to consider modifiable factors affecting patient adherence and satisfaction when optimising individual treatment strategies. However, such factors have not been well studied in Japan. Furthermore, factors affecting patient adherence and satisfaction may differ between relatively young and elderly patients due to their different socioeconomical, physio-psychological and financial backgrounds. Three-quarters of people in Japan who were 'suspected of having diabetes' - based on a reported $\mathrm{HbA} 1 \mathrm{c}$ value of $\geq 6.5 \%$ or because they were under diabetes treatment-were aged $\geq 65$ years according to a national survey in 2017 [32]. Yet, few studies have focused on investigating age-specific factors affecting patient adherence and satisfaction.

To identify modifiable factors associated with treatment adherence and satisfaction, we conducted a web-based questionnaire on factors encompassing patient demographics, disease and physiopsychological status, the impact of receiving/understanding physician guidance on treatment goals and diabetes management, and socioeconomic status. Furthermore, we stratified the factors by age group ( $<65$ or $\geq 65$ years) to understand how their impact might differ between young and elderly patients.

\section{METHODS}

\section{Study Design and Setting}

This study is an analysis of responses to a webbased questionnaire survey conducted from 6 to 17 March 2019 in Japan. Survey participants were recruited from registered members of research panels (one general and one disease focused) operated by Cross Marketing, Inc. (Tokyo, Japan). Those who consented to participate in the survey and reported having seen a doctor or had been admitted to a hospital for treatment of T2DM within the year prior to January 2019 were invited to the survey via email. The questionnaire was developed by the authors (including a JDS-certified diabetologist) in collaboration with Social Survey Research Information Co. Ltd. (SSRI; Tokyo, Japan), with the aim of surveying three areas of research interest: (1) factors associated with T2DM treatment adherence and satisfaction (the present study); (2) factors associated with treatment continuation/discontinuation and the relationship between shared decision making, T2DM treatment satisfaction and continuation rate; (3) circumstances surrounding clinical inertia in T2DM treatment. The questionnaire was evaluated and revised by JDS-certified diabetologists among the authors to ensure that the expressions were appropriate. The order of answer options was randomised where appropriate to manage multiple-choice answer option order bias. The questionnaire comprised eight screening and 39 survey questions that required single-choice, multiple-choice or numeric answers, was designed to be completed within $15 \mathrm{~min}$, and was written in Japanese (an English version of the questionnaire is provided in the Supplementary Information). The protocol for this study was registered at the Japan Pharmaceutical Information Center (JapicCTI-194636). Factors associated with T2DM treatment adherence and satisfaction were assessed in the overall patient population and in subgroups separated by age ( $<65$ or $\geq 65$ years). Factors associated with adherence in working patients with T2DM were further assessed.

\section{Participants and Study Size}

Inclusion criteria for participation in the study were an age of 20 years or older, a diagnosis of T2DM, not a healthcare provider, and seeing a doctor and receiving medication for treatment of T2DM. The exclusion criterion was the provision of inconsistent, erroneous or inappropriate 
responses. All excluded cases were documented with justifications. The age composition of the target study population was designed to match that of people in Japan who are highly suspected to have diabetes (based on a self-declared HbA1c value of $\geq 6.5 \%$ or because they were receiving treatment for diabetes), as reported in the National Health and Nutrition Survey by the Japanese Ministry of Health, Labour and Welfare [32]. Once the target proportion of predefined age groups (20-39 years, $4 \%$; 40-49 years, $10 \%$; 5059 years, $16 \% ; \quad 60-69$ years, $27 \%$; $\geq 70$ years, $42 \%)$ was reached, subsequent respondents were excluded from the analysis. We aimed to collect 1,000 independent analysable questionnaire survey samples to ensure the enrolment of a sufficient number of patients in the 20-39 year age group. Collection of survey data was completed when the target number of patients in each age group was reached.

\section{Statistical Analysis and Software}

Survey response data were tabulated using the Hideyoshi Dplus software (SSRI, Tokyo, Japan) and stored in Excel files. Treatment adherence was assessed in survey question Q11 (What percentage of the time do you take your antidiabetic drugs (including injections) as specified (timing, number of times)? Answer between $0 \%$ (I never take it) and $100 \%$ (I always take it); see the Supplementary Information); patients who entered a value of $\geq 80 \%$ were deemed as being adherent to their treatment, whereas patients were deemed as being nonadherent when they entered a value of $<80 \%$. Treatment satisfaction was evaluated on a five-point Likert scale ranging from "mostly satisfied" to "mostly dissatisfied". Participants who answered "mostly satisfied" or "somewhat satisfied" to survey question Q22 (Tell us how satisfied you are with your current diabetes treatment; see the Supplementary Information) were deemed as being being satisfied with their treatment, and whereas they were deemed as being unsatisfied when the answers were selected from the remaining three options (scale ranging from "neither satisfied nor dissatisfied" to "mostly dissatisfied"). Outcomes from twenty additional questions surveying factors encompassing training, education, understanding of treatment goals and diabetes management, and socioeconomic status (S1-S3, Q1, Q3, Q21, Q22, Q28-Q36; see the Supplementary Information) were binarised (groupings of the answer options are shown in Tables 2 and 3; see "Variable" and "Reference"), except for age and disease duration. The outcome data were then used as independent variables in a multiple logistic regression model after testing for multicollinearity (the cutoff for the pairwise correlation coefficient was 0.8 ), with treatment adherence or satisfaction as the dependent variable. The adjusted odds ratio (OR) and the two-sided 95\% confidence interval (CI) for each factor were calculated and tested using the Wald test with a significance level of $5 \%$ in BellCurve for Excel version 3.10 (SSRI, Tokyo, Japan).

The chi-square test was used with a significance level of $5 \%$ to examine the factors affecting treatment adherence in patients who were employed. Where multiple comparisons were made against the same group of patients, the $p$-values were adjusted to correct for multiple hypothesis testing using the false discovery rate method [33]. When at least one cell of the contingency table had an expected frequency smaller than 5, the $p$-values were adjusted using Yates's correction.

To ensure data quality, SSRI was employed to check the data independently of the authors using Excel functions to locate any inconsistencies, errors or malicious answers.

\section{Ethics Compliance}

This study was conducted in compliance with the Ethical Guidelines for Medical and Health Research Involving Human Subjects and their guidance $[33,34]$, and in accordance with the ethical principles of the 1964 Declaration of Helsinki and the International Conference on Harmonisation Guideline for Good Clinical Practice. The study was reviewed and approved by the ethics committee at the Research Institute of Healthcare Data Science, Tokyo (receipt no. RI2018017). All participants consented to participate in the survey and could discontinue the survey at any time. All responses were 
anonymised at the time of data collection in an unlinkable manner.

\section{RESULTS}

\section{Participants}

Of the 1,124 survey respondents, 1,000 were included in the analysis. Of the 124 excluded responses, 109 were excluded to match to the age distribution of patients with T2DM in Japan [32], 9 were excluded because the time spent completing the survey was deemed too short $(<4 \mathrm{~min}), 5$ were excluded because the respondent gave the same answer for multiple questions, and 1 was excluded because of an inappropriate answer given for Q15 (Tell us the main reasons why you would be reluctant to intensify your diabetes treatment; see the Supplementary Information).

\section{Patient Characteristics}

According to the completed survey forms, the mean age of the analysed patients was 63.8 years, $73.9 \%$ were male, and the mean age of initial diagnosis was 50.1 years (Table 1). Patients received care at clinics $(59.5 \%)$ or at hospitals (40.5\%). The most frequent comorbidities reported by the patients were hypertension $(44.5 \%)$, dyslipidaemia (21.0\%) and obesity (12.9\%); the most common complications were diabetic retinopathy $(8.3 \%)$, diabetic neuropathy $(7.7 \%)$, and myocardial infraction (7.7\%).

\section{Treatment Adherence and Satisfaction}

In the overall population, 941 (94.1\%) and 575 (57.5\%) patients were classified as adherent and satisfied, respectively. Of the participants aged $\geq 65$ and $<65$ years, treatment adherence was evident in $96.9 \%$ and $90.4 \%$, and treatment satisfaction in $62.9 \%$ and $50.4 \%$, respectively.

\section{Factors Affecting Treatment Adherence}

In the overall population, treatment adherence was positively associated with increased age (OR
Table 1 Patient characteristics

\begin{tabular}{|c|c|}
\hline Patient characteristic & $(N=1000)$ \\
\hline Age (years), mean $\pm S D$ & $63.8 \pm 11.9$ \\
\hline $\begin{array}{l}\text { When diabetes was indicated/suspected } \\
\text { from test results (years), mean } \pm S D\end{array}$ & $49.2 \pm 11.9$ \\
\hline $\begin{array}{l}\text { When the patient first saw a doctor for } \\
\text { their diabetes (years), mean } \pm S D\end{array}$ & $50.1 \pm 11.7$ \\
\hline Duration of diabetes ${ }^{\mathrm{a}}$ (years), mean $\pm S D$ & $14.6 \pm 9.9$ \\
\hline Male & $739(73.9)$ \\
\hline Employed & $398(39.8)$ \\
\hline \multicolumn{2}{|l|}{ Diabetes care is primarily received at } \\
\hline Clinic & $595(59.5)$ \\
\hline Hospital & $405(40.5)$ \\
\hline \multicolumn{2}{|l|}{ Type of clinical department: } \\
\hline Diabetes/metabolic endocrinology & $388(38.8)$ \\
\hline General internal medicine & $570(57.0)$ \\
\hline Other & $42(4.2)$ \\
\hline \multicolumn{2}{|l|}{ Comorbidities } \\
\hline Hypertension & $445(44.5)$ \\
\hline Dyslipidaemia & $210(21.0)$ \\
\hline Obesity/metabolic syndrome & $129(12.9)$ \\
\hline Mental illness & $63(6.3)$ \\
\hline Kidney disease & $13(1.3)$ \\
\hline \multicolumn{2}{|l|}{ Diabetic complications } \\
\hline Diabetic retinopathy & $83(8.3)$ \\
\hline Myocardial infarction & $77(7.7)$ \\
\hline Diabetic neuropathy & $77(7.7)$ \\
\hline Diabetic nephropathy & $40(4.0)$ \\
\hline Stroke & $23(2.3)$ \\
\hline Arteriosclerosis obliterans & $12(1.2)$ \\
\hline $\begin{array}{l}\text { Highest level of educational attainment } \\
\text { (university) }\end{array}$ & $467(46.7)$ \\
\hline \multicolumn{2}{|l|}{ Household composition } \\
\hline Living with spouse/partner & $447(44.7)$ \\
\hline Two generations & $325(32.5)$ \\
\hline
\end{tabular}


Table 1 continued

\begin{tabular}{|c|c|}
\hline Patient characteristic & $(N=1000)$ \\
\hline Single & $161(16.1)$ \\
\hline Three generations & $49(4.9)$ \\
\hline Others & $18(1.8)$ \\
\hline \multicolumn{2}{|c|}{$\begin{array}{l}\text { Understanding the doctor's explanation } \\
\text { regarding: }\end{array}$} \\
\hline \multicolumn{2}{|c|}{$\begin{array}{l}\text { The goal of achieving the HbAlc target } \\
\text { level }\end{array}$} \\
\hline Understood & $819(81.9)$ \\
\hline Did not understand & $85(8.5)$ \\
\hline Not explained & $96(9.6)$ \\
\hline \multicolumn{2}{|l|}{ Diabetes } \\
\hline Understood & $827(82.7)$ \\
\hline Did not understand & $86(8.6)$ \\
\hline Not explained & $87(8.7)$ \\
\hline \multicolumn{2}{|l|}{ Diabetic complications } \\
\hline Understood & $864(86.4)$ \\
\hline Did not understand & $75(7.5)$ \\
\hline Not explained & $61(6.1)$ \\
\hline \multicolumn{2}{|l|}{ Nutrition therapy } \\
\hline Understood & $746(74.6)$ \\
\hline Did not understand & $120(12.0)$ \\
\hline Not explained & $134(13.4)$ \\
\hline \multicolumn{2}{|l|}{ Exercise therapy } \\
\hline Understood & $679(67.9)$ \\
\hline Did not understand & $113(11.3)$ \\
\hline Not explained & $208(20.8)$ \\
\hline \multicolumn{2}{|c|}{ Tailoring of pharmacologic therapy } \\
\hline Understood & $710(71.0)$ \\
\hline Did not understand & $162(16.2)$ \\
\hline
\end{tabular}

Table 1 continued

\begin{tabular}{l}
\hline Patient characteristic $\quad(\boldsymbol{N}=\mathbf{1 0 0 0})$ \\
\hline Not explained \\
\hline Values show are $n(\%)$ unless otherwise indicated \\
${ }^{a}$ Calculated as the difference between the current age and \\
the age when diabetes was indicated/suspected from test \\
results \\
$S D$ standard deviation
\end{tabular}

$1.04,95 \%$ CI 1.02-1.07; $p=0.002$ ) and a household income of $\geq 4$ million Japanese yen (JPY) (OR 2.07, 95\% CI 1.11-3.86; $p=0.022$ ) (Table 2). Being employed (OR 0.30, 95\% CI $0.15-0.60 ; p=0.001$ ) and having $\geq 1$ impaired basic activity of daily living (BADL) (OR 0.33, 95\% CI $0.13-0.82 ; p=0.017$ ) were negatively associated with treatment adherence. In patients $<65$ years, treatment adherence was positively associated with a household income of $\geq 4$ million JPY (OR 2.37, 95\% CI 1.09-5.16; $p=0.029$ ), and was negatively associated with having difficulties with $\geq 1$ impaired BADL (OR 0.27 , 95\% CI 0.08-0.92; $p=0.036$ ). Employment was negatively correlated with treatment adherence in both age groups: patients aged $\geq 65$ (OR 0.31, 95\% CI 0.10-0.95; $p=0.040$ ) and $<65$ years (OR 0.27, 95\% CI $0.10-0.70$; $p=0.007)$. A positive, though nonsignificant, association between treatment adherence and satisfaction was also observed in the overall population (OR 1.72, 95\% CI 0.94-3.14; $p=0.079$ ).

\section{Factors Affecting Treatment Satisfaction}

A multiple logistic regression model was used to assess the impact of parameters encompassing patient demographics, disease status, understanding of treatment goals and diabetes management, and socioeconomic status on treatment satisfaction (Table 3). In the overall patient population, treatment satisfaction was positively associated with receiving and understanding guidance on how pharmacologic therapies are tailored (OR 1.73, 95\% CI $1.19-2.51 ; p=0.004)$, male sex (OR 1.55, 95\% CI 1.10-2.19; $p=0.013)$, a household income of 


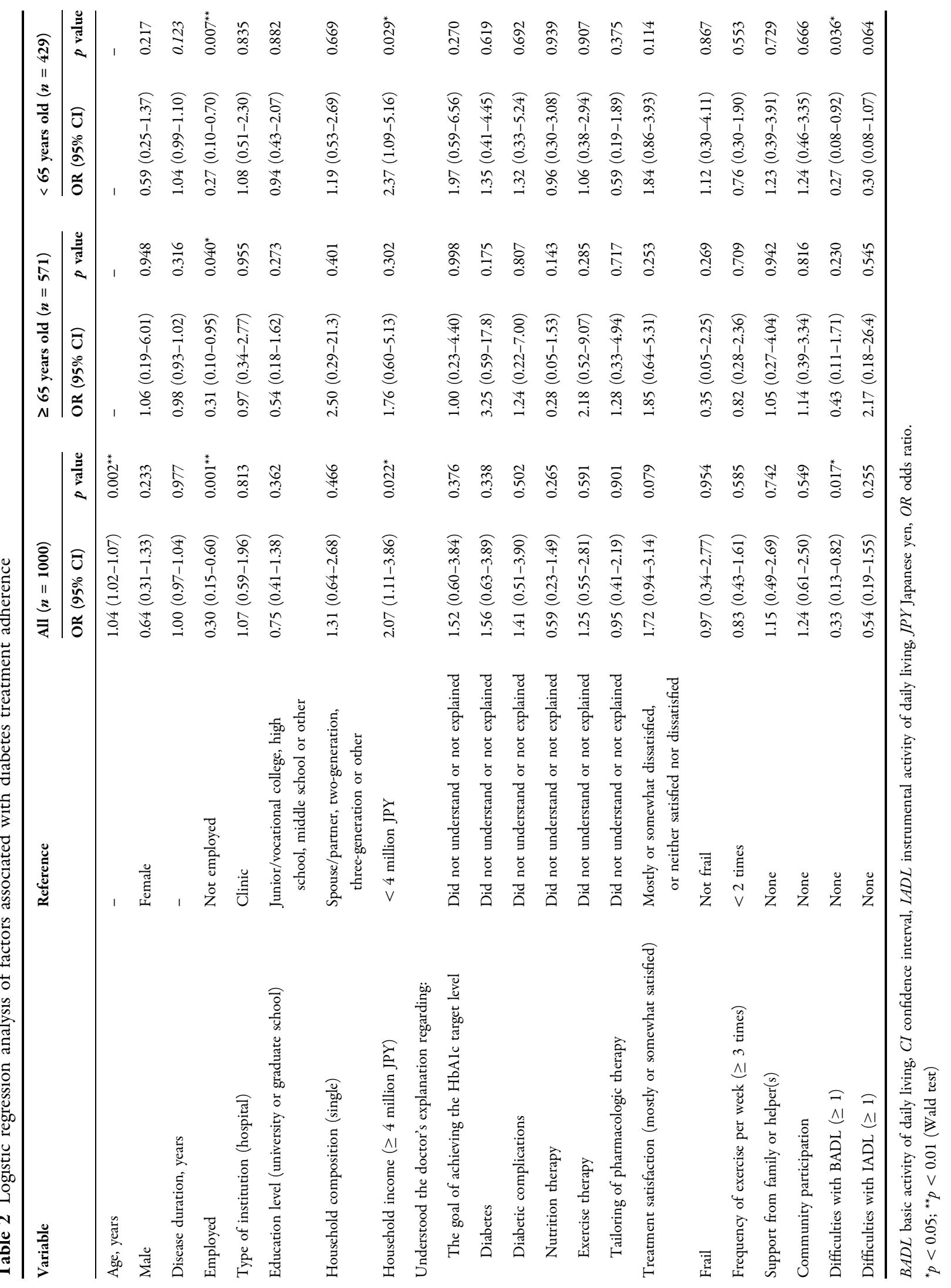




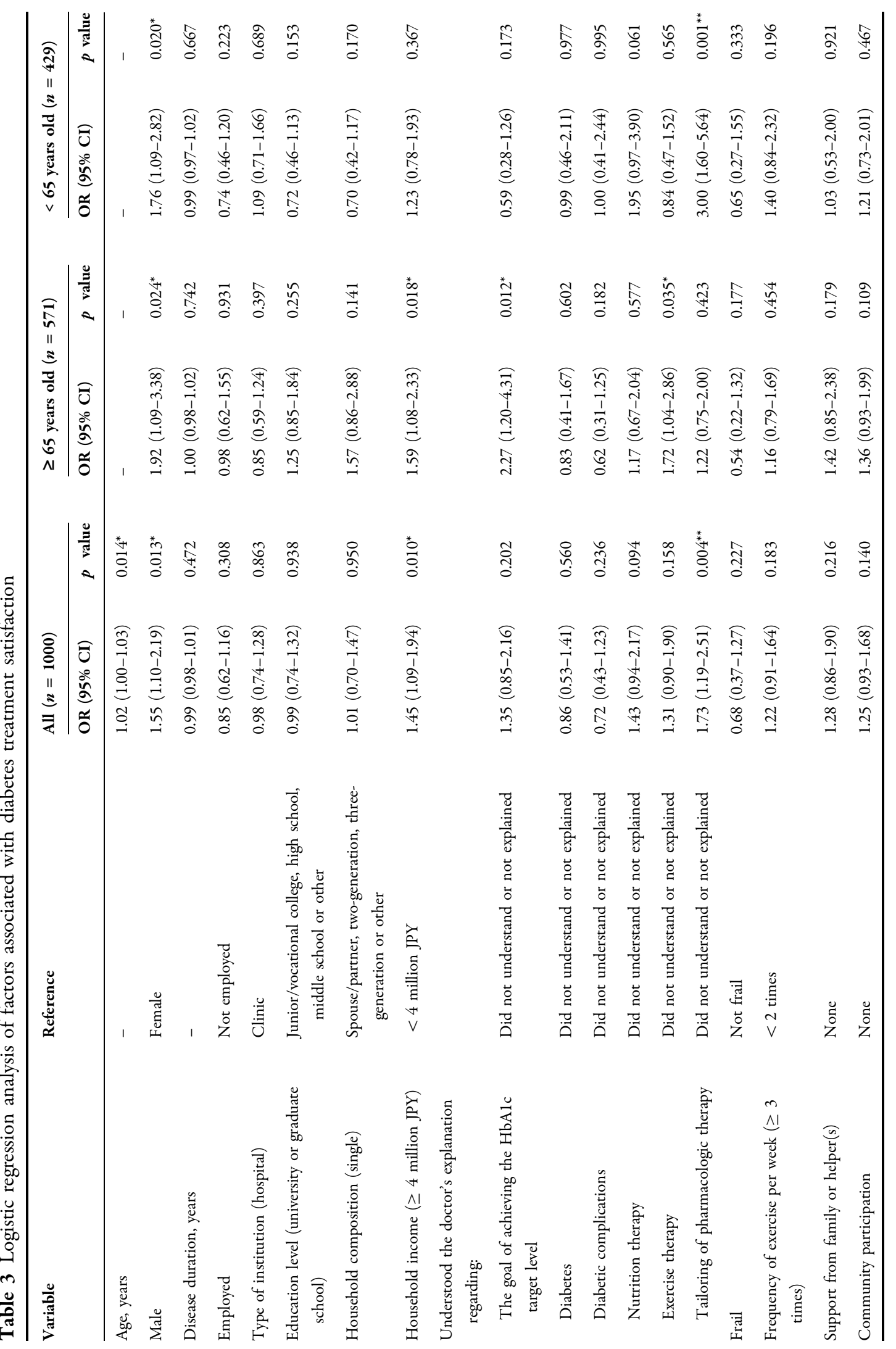




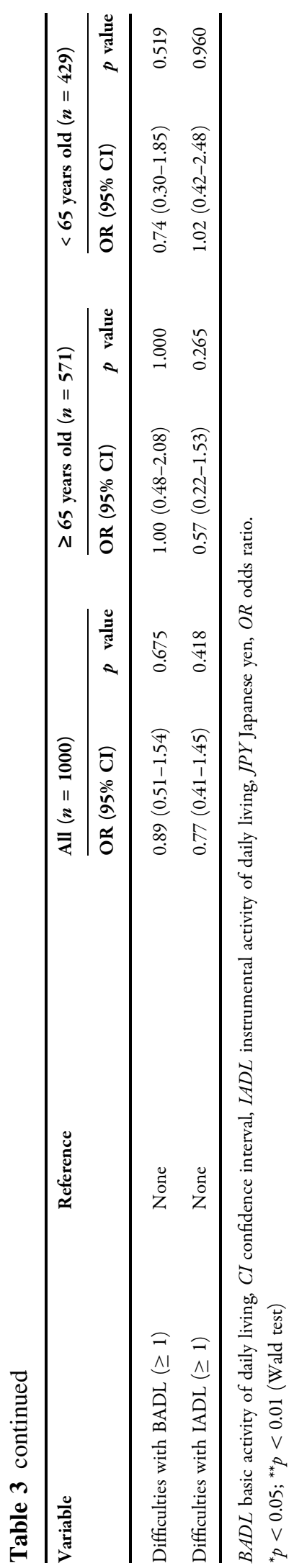

ver 4 million JPY (OR 1.45, 95\% CI 1.09-1.94; $p=0.010)$ and increased age (OR 1.02, 95\% CI $1.00-1.03 ; p=0.014)$. Most of the factors affecting treatment satisfaction in patients aged $\geq 65$ years differed from the factors affecting treatment satisfaction in patients aged $<65$ years; the former included receiving and understanding guidance on the importance of achieving target HbA1c values (OR 2.27, 95\% CI $1.20-4.31 ; p=0.012$ ), receiving and understanding guidance on exercise therapies (OR $1.72,95 \%$ CI 1.04-2.86; $p=0.035)$ and a household income of 4 million JPY or more (OR $1.59,95 \%$ CI 1.08-2.33; $p=0.018$ ). In patients aged $<65$ years, treatment satisfaction was significantly higher in those who received and understood guidance on the tailoring of pharmacologic therapies (OR 3.00, 95\% CI $1.60-5.64 ; p=0.001)$. Males were significantly more satisfied with their treatment than females in patients aged $\geq 65$ (OR 1.92, 95\% CI $1.09-3.38 ; p=0.024)$ and $<65$ years (OR 1.76 , 95\% CI 1.09-2.82; $p=0.020)$.

\section{Employment and Treatment Adherence}

The relationship between treatment adherence and patient employment was tested using the chi-square test, revealing a significant negative association between employment and treatment adherence $(p=0.001$, Fig. 1$)$. The proportions of adherent patients were $97.0 \%$ and $89.7 \%$ among those who were unemployed and employed, respectively. To explore factors affecting treatment adherence in patients who were employed, we used the chi-square test to examine the relationships between other parameters and treatment adherence (Fig. 2); among employed patients, adherence decreased as age decreased (20-39 years vs $\geq 70$ years; $p=0.0011$ ) and as the number of family generations living together increased (partner only vs two generations $[p=0.0053]$, partner only vs three generations $[p=0.0102])$. Treatment adherence in employed patients was positively associated with a household income of $\geq 4$ million JPY $(p=0.0328)$ and treatment satisfaction $(p=0.0354)$, and was negatively associated with having $\geq 1$ impaired BADL 


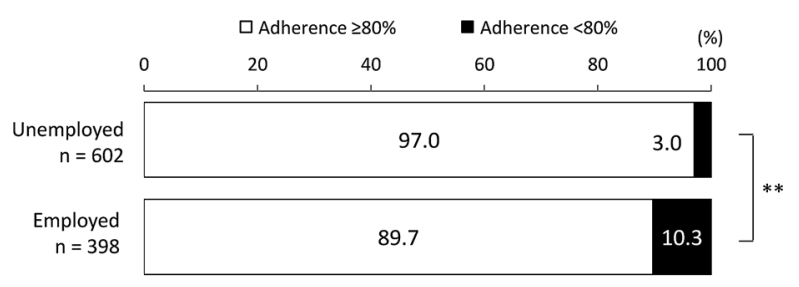

Fig. 1 Association between adherence and employment. ${ }^{* *} p<0.01$ (chi-square test)

$(p=0.0002)$, having $\geq 1$ impaired instrumental activity of daily living (IADL) $(p=3.0 \mathrm{e}-6)$ and having been on an educational hospital admission programme for diabetes $(p=0.0018)$. Treatment adherence was negatively associated with having diabetic neuropathy $(p=0.0005)$. All other factors tested showed no significant association with treatment adherence (see Fig. S1 in the Supplementary Information).

\section{DISCUSSION}

Factors affecting diabetes treatment adherence and satisfaction have been extensively studied worldwide; however, studies in Japan have been limited. The present study explored potential factors affecting treatment adherence and satisfaction in T2DM management in Japan using a web-based patient survey. Our analysis examined differences in factors between patients aged $\geq 65$ and $<65$ years, and also sought to investigate the impact of impairment in activities of daily living (ADLs) on T2DM treatment adherence and satisfaction for the first time, to our knowledge.

\section{Factors Affecting T2DM Treatment Adherence in Japan}

Positive factors associated with treatment adherence in patients with T2DM in Japan were higher age and household income; these findings are consistent with previous studies in the United States $[15,16]$. A higher proportion of satisfied patients were adherent compared with unsatisfied patients, though the OR did not reach statistical significance.

Our study shows that employed patients were three times less likely to be adherent with their treatment compared with unemployed patients. This result is consistent with the findings of the Japan Diabetes Outcome Intervention Trial 2 (J-DOIT2), a nationwide largescale survey investigating reasons for treatment discontinuation in patients with T2DM [20]. In J-DOIT2, reported reasons for treatment discontinuation included (1) a lack of awareness of the importance of prioritising treatment (citing other priorities, including work, school and family); (2) not recognising the need to receive treatment (citing not seeing the doctor because the patient was feeling well); and (3) the financial burden of medical expense. These findings underscore the importance of implementing targeted interventions to promote treatment adherence in working patients with T2DM in Japan.

Having difficulties with $\geq 1$ BADL was negatively associated with treatment adherence in the overall population and in patients aged $<65$ years. The presence of impaired BADL and IADL is considered when setting a target HbA1c level for elderly patients [3, 4]; however, to our knowledge, the impact of ADLs on treatment adherence has not been reported. Further investigation is warranted to clarify the relationship between treatment adherence and impaired BADL, especially in patients with $\mathrm{T} 2 \mathrm{DM}$ aged $<65$ years, and to identify potential interventions to address the reduced adherence.

\section{Factors Affecting T2DM Treatment Satisfaction in Japan}

Two previous studies have reported that adherence to treatment and perceived hypoglycaemia are factors associated with treatment satisfaction in patients with T2DM in urban districts in 


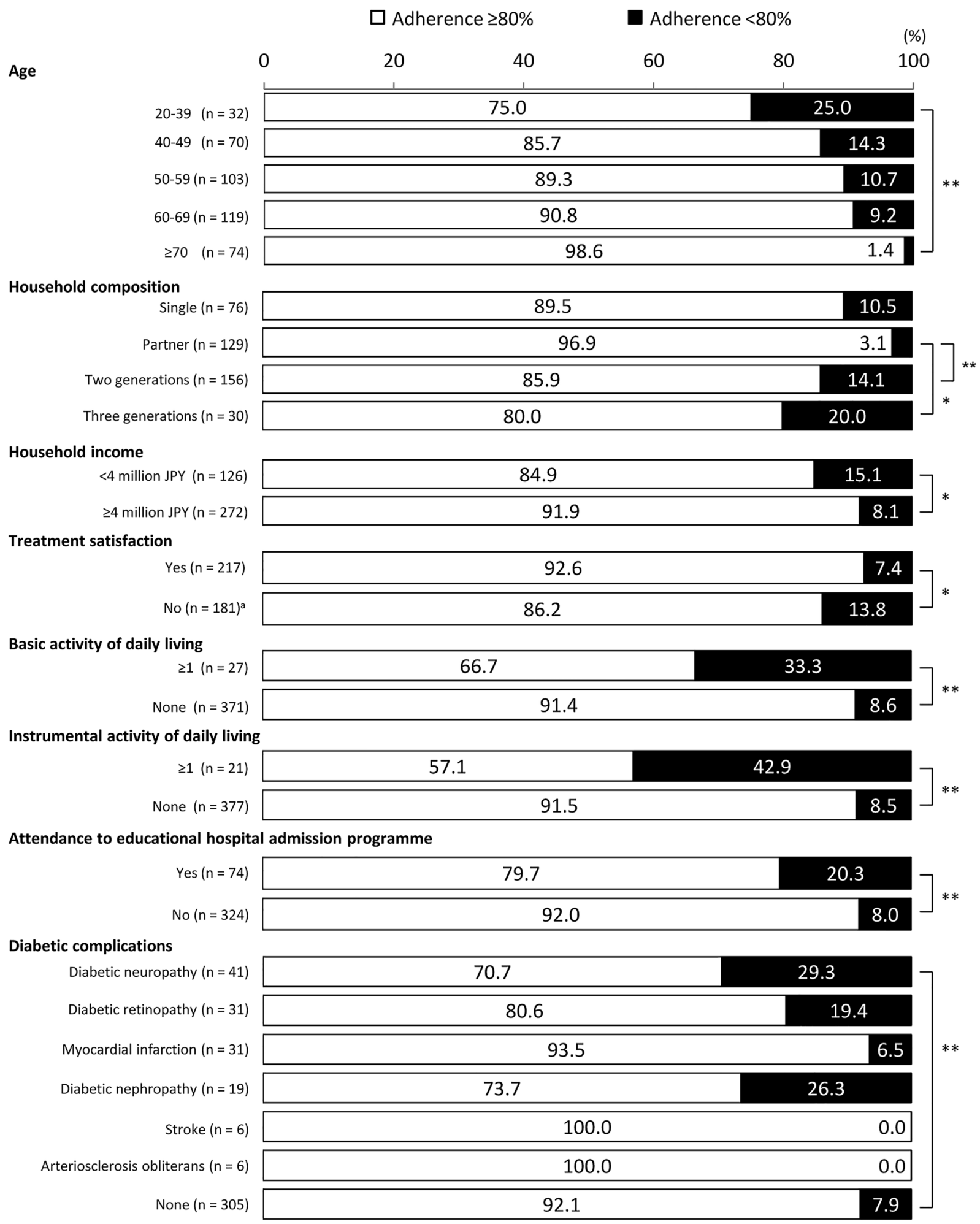

Fig. 2 Factors associated with treatment adherence in T2DM patients who are employed. ${ }^{*} p<0.05 ;{ }^{* *} p<0.01$ (chi-square test). ${ }^{a}$ Includes those who are neither satisfied nor dissatisfied 
Japan [21, 22]. Some of the factors significantly associated with treatment satisfaction in our study were not found to be significant in those previous Japanese studies, possibly due to the difference in the study population; our study enrolled patients across the nation who were registered in panels operated by Cross Marketing, Inc., and the age distribution of the patients was matched to that of patients with T2DM in Japan according to a national survey [32]. Our analysis identified factors that have been reported as significant in studies from multiple other countries in Europe, North America and the Middle East; these include increased age [30], male sex [23, 26] and higher income [28]. A study from Qatar reported an association between lower treatment satisfaction and increased age [27]; this suggests that age may impact treatment satisfaction in different countries differently.

Patients in the overall patient population and among those aged $<65$ years who received and understood guidance on the tailoring of pharmacologic therapies from their physician were 1.7 and 3 times more likely to be satisfied with their treatment, respectively, but patients aged $\geq 65$ years who received and understood such guidance were not significantly more satisfied (Table 2). These results suggest that a better patient understanding of how pharmacologic therapies are tailored may improve treatment satisfaction, particularly in patients with T2DM aged $<65$ years. Most patients aged $\geq 65$ years may have been receiving pharmacologic therapies for a long enough time to understand how their medications are selected and to establish a habit of taking them, so guidance may have less of an impact for those patients. On the other hand, guidance on the importance of achieving the target HbA1c for maintaining normal QoL and life expectancy, and on the importance of exercise therapy, did increase the odds of being satisfied with the treatment in patients aged $\geq 65$ years. To our knowledge, this is the first report on modifiable factors specifically associated with treatment satisfaction in elderly patients with T2DM. Elderly patients may be more mindful about spending sufficient time on health maintenance and exercise than working patients aged $<65$ years are, which would explain the stronger impact of guidance on exercise therapy in the elderly patients.

\section{Treatment Adherence in Working Patients with T2DM in Japan}

Among working patients with T2DM, a correlation between younger age and poor adherence was evident, as in the overall population. These results suggest that it is important to implement support initiatives targeting younger patients in Japan. Employed patients who had difficulties in $\geq 1 \mathrm{ADL}$ were less frequently adherent compared with those who did not, and employed patients living with multiple family generations (e.g., children and/or parents) were less frequently adherent compared with those living with their spouse or partner only. Further investigation into strategies to neutralise the negative impact of these factors may help improve treatment adherence in these groups. Employed patients who participated in an educational hospital admission programme for diabetes were less frequently adherent than those who had not; poor adherence may have led to poor glycaemic control, which may have resulted in the prescription of educational hospital admissions. Interestingly, a recent study in Japan has reported that patients who visited the doctor's office $\geq 17$ times over the past 3 years were 24 times more likely to be adherent to treatment (vs patients with $<17$ visits) [17]. Thus, frequent guidance, communication, and prompts to visit a clinic may play an important role in promoting treatment adherence in young employed patients. Finally, low treatment satisfaction was associated with low treatment adherence in employed patients, suggesting that improving treatment satisfaction may improve adherence in employed patients with T2DM.

\section{Limitations and Strengths}

This study was an uncontrolled observational study, and patients were limited to those who were registered in research panels operated by 
Cross Marketing, Inc. and agreed to participate in the survey. Sampling bias may be present because of the nature of web surveys; however, the age composition of the target study population was designed to match that of patients with T2DM in Japan [32] to minimise potential bias. The analysis was based on the participants' self-assessment and is limited by the lack of objectivity and the potential for inaccuracies. Further investigations are needed to validate the findings of this study. A strength of this study is that the questionnaire was designed to provide a detailed account of the patients' own perspective on diabetes treatment adherence and satisfaction.

\section{CONCLUSIONS}

Lower age, female sex, lower household income and not receiving or understanding physician guidance on the tailoring of pharmacologic therapies may negatively impact treatment satisfaction. Lower age, lower household income, employment and impaired BADL may negatively impact treatment adherence. Appropriate physician guidance and improved management/prevention of ADL disability may be effective in improving T2DM treatment adherence or satisfaction. Factors significantly associated with T2DM treatment adherence and satisfaction differed between patients aged $<65$ and those aged $\geq 65$ years, so such differences should be considered in intervention strategies. The results provide valuable insights into the factors affecting treatment adherence and treatment satisfaction in the management of T2DM in Japan.

\section{ACKNOWLEDGEMENTS}

We are grateful to the patients who participated in this study. We thank Eri Nakajima, Masato Oosawa and Yasushi Kawakita of Takeda Pharmaceutical Company Limited for their support during the planning and designing of the survey. The survey data was managed and analysed by Kohei Mizui and Chie Kikutake of Social Survey Research Information Co. Ltd.
Funding. Takeda Pharmaceutical Company Limited (Tokyo, Japan) funded this study, assisted with the preparation of this manuscript, and will sponsor the journal's Rapid Service Fee.

Medical Writing and Editorial Assistance. Manuscript development was assisted by Fumiko Shimizu of MIMS Co. Ltd., funded by Takeda Pharmaceutical Company Ltd., in compliance with Good Publication Practice 3 ethical guidelines (Battisti et al., Ann Intern Med 2015;163:461-4).

Authorship. All named authors meet the International Committee of Medical Journal Editors (ICMJE) criteria for authorship for this article, take responsibility for the integrity of the work as a whole, and have given their approval for this version to be published.

Authors' Contributions. All authors contributed to the conception of the study design, data analysis and interpretation, and revising the manuscript for important intellectual content. SS, NN, KK, TM contributed to drafting of the manuscript.

Disclosures. Ryo Suzuki reports personal fees from Takeda Pharmaceutical Co., Ltd. during the conduct of the study; grants and personal fees from MSD K.K., grants from Daiichi Sankyo Co., Ltd., personal fees from Novartis Pharma K.K., Takeda Pharmaceutical Co., Ltd., Mitsubishi Tanabe Pharma Corporation, Sanofi K.K., Novo Nordisk Pharma Ltd., Sumitomo Dainippon Pharma Co., Ltd., Eli Lilly Japan K.K., Ono Pharmaceutical Co., Ltd., outside the submitted work. Shun Saita, Nobuhiro Nishigaki, Koichi Kisanuki, Yukiko Shimasaki and Tomoko Mineyama are employed by Takeda Pharmaceutical Company Limited which funded this study. Masato Odawara reports personal fees from Takeda Pharmaceutical Co., Ltd., during the conduct of the study; personal fees from MSD K.K., Novartis Pharma K.K., Taisho Pharmaceutical Co., Ltd., grants and personal fees from Daiichi Sankyo Co., Ltd., Takeda Pharmaceutical Co., Ltd., Mitsubishi Tanabe Pharma Corporation, Sumitomo 
Dainippon Pharma Co., Ltd., Eli Lilly Japan K.K., Ono Pharmaceutical Co., Ltd., Astellas Pharma Inc., Kyowa Kirin Co., Ltd., Nippon Boehringer Ingelheim Co., Ltd. outside the submitted work. Current affiliations: Yukiko Shimasaki, Nihonbashi Cardiology Clinic (Tokyo, Japan); and Tomoko Mineyama, Department of Endocrinology and Metabolism, Tama-Hokubu Medical Center (Tokyo, Japan).

Compliance with Ethics Guidelines. This study was conducted in compliance with the Ethical guidelines for Medical and Health Research Involving Human Subjects and their guidance $[33,34]$, and in accordance with the ethical principles of the 1964 Declaration of Helsinki and the International Conference on Harmonisation Guideline for Good Clinical Practice. The study was reviewed and approved by ethics committee in the Research Institute of Healthcare Data Science, Tokyo (receipt No. RI2018017). All participants consented to participate in the survey and could discontinue the survey at any time. All responses were anonymised at the time of data collection in an unlinkable manner.

Data Availability. The datasets generated during and/or analyzed during the current study are available from the corresponding author on reasonable request. The datasets, including the redacted study protocol, redacted statistical analysis plan, and individual participants data supporting the results reported in this article, will be available three months from initial request, to researchers who provide a methodologically sound proposal. The data will be provided after its de-identification, in compliance with applicable privacy laws, data protection and requirements for consent and anonymisation.

Open Access. This article is licensed under a Creative Commons Attribution-NonCommercial 4.0 International License, which permits any non-commercial use, sharing, adaptation, distribution and reproduction in any medium or format, as long as you give appropriate credit to the original author(s) and the source, provide a link to the Creative Commons licence, and indicate if changes were made. The images or other third party material in this article are included in the article's Creative Commons licence, unless indicated otherwise in a credit line to the material. If material is not included in the article's Creative Commons licence and your intended use is not permitted by statutory regulation or exceeds the permitted use, you will need to obtain permission directly from the copyright holder. To view a copy of this licence, visit http://creativecommons.org/licenses/by$\mathrm{nc} / 4.0 /$.

\section{REFERENCES}

1. Hara K, Kadowaki T, Odawara M. Genes associated with diabetes: potential for novel therapeutic targets? Expert Opin Ther Targets. 2016;20(3):255-67.

2. Kaku K. Pathophysiology of type 2 diabetes and its treatment policy. JMAJ. 2010;53(1):41-6.

3. American Diabetes Association. Standards of medical care in diabetes 2019. Diabetes Care. 2019;42: S1-193.

4. Araki E, Goto A, Kondo T, Noda M, Noto H, Origasa $\mathrm{H}$, et al. Japanese clinical practice guideline for diabetes 2019. Diabetol Int. 2020;11(3):165-223.

5. Yokoyama H, Oishi M, Takamura H, Yamasaki K, Shirabe SI, Uchida D, et al. Large-scale survey of rates of achieving targets for blood glucose, blood pressure, and lipids and prevalence of complications in type 2 diabetes (JDDM 40). BMJ Open Diabetes Res Care. 2016;4(1):e000294.

6. Stratton IM, Adler AI, Neil HA, Matthews DR, Manley SE, Cull CA, et al. Association of glycaemia with macrovascular and microvascular complications of type 2 diabetes (UKPDS 35): prospective observational study. BMJ. 2000;321(7258):405-12.

7. Diabetes C, Complications Trial Research G, Nathan DM, Genuth S, Lachin J, Cleary P, et al. The effect of intensive treatment of diabetes on the development and progression of long-term complications in insulin-dependent diabetes mellitus. N Engl J Med. 1993;329(14):977-86.

8. The Japan Diabetes Society and Japan Association for Diabetes Education and Care aim to create a society where patients do not need to hide their diabetes. Diabetes Mellitus Resource Guide. Tokyo, 
Japan: Soshinsha Co., Ltd, 2019. http://dm-rg.net/ news/2019/11/020214.html?pr=dmrg001. Accessed Mar 2020.

9. Japan Diabetes Clinical Data Management Study Group. Basic statistics. Ibaraki, Japan: Japan Diabetes Clinical Data Management Study Group; 2019. http://jddm.jp/data/index-2019/.

10. Wagner EH, Sandhu N, Newton KM, McCulloch DK, Ramsey SD, Grothaus LC. Effect of improved glycemic control on health care costs and utilization. JAMA. 2001;285(2):182-9.

11. Egede LE, Gebregziabher M, Echols C, Lynch CP. Longitudinal effects of medication nonadherence on glycemic control. Ann Pharmacother. 2014;48(5):562-70.

12. Rozenfeld Y, Hunt JS, Plauschinat C, Wong KS. Oral antidiabetic medication adherence and glycemic control in managed care. Am J Manag Care. 2008;14(2):71-5.

13. Krapek K, King K, Warren SS, George KG, Caputo DA, Mihelich K, et al. Medication adherence and associated hemoglobin A1c in type 2 diabetes. Ann Pharmacother. 2004;38(9):1357-62.

14. Krass I, Schieback P, Dhippayom T. Adherence to diabetes medication: a systematic review. Diabet Med. 2015;32(6):725-37.

15. Kirkman MS, Rowan-Martin MT, Levin R, Fonseca VA, Schmittdiel JA, Herman WH, et al. Determinants of adherence to diabetes medications: findings from a large pharmacy claims database. Diabetes Care. 2015;38(4):604-9.

16. Curkendall SM, Thomas N, Bell KF, Juneau PL, Weiss AJ. Predictors of medication adherence in patients with type 2 diabetes mellitus. Curr Med Res Opin. 2013;29(10):1275-86.

17. Horii T, Momo K, Yasu T, Kabeya Y, Atsuda K. Determination of factors affecting medication adherence in type 2 diabetes mellitus patients using a nationwide claim-based database in Japan. PLoS ONE. 2019;14(10):e0223431.

18. Shams N, Amjad S, Kumar N, Ahmed W, Saleem F. Drug non-adherence in type 2 diabetes mellitus; predictors and associations. J Ayub Med Coll Abbottabad. 2016;28(2):302-7.

19. Farhat R, Assaf J, Jabbour H, Licha H, Hajj A, Hallit $\mathrm{S}$, et al. Adherence to oral glucose lowering drugs, quality of life, treatment satisfaction and illness perception: a cross-sectional study in patients with type 2 diabetes. Saudi Pharm J. 2019;27(1):126-32.
20. Japan Human Data Society of Diabetes and Related Diseases. Japanese practice guidance summary to improve patients' adherence to appointments for diabetes care. Tokyo, Japan: Japan Human Data Society of Diabetes and Related Diseases; 2014. Accessed May 2014. http://human-data.or.jp/wp/ wp-content/uploads/2018/07/dm_jushinchudan_ manual_e.pdf.

21. Saisho Y. Use of diabetes treatment satisfaction questionnaire in diabetes care: importance of patient-reported outcomes. Int J Environ Res Public Health. 2018;15(5):947.

22. Hayashi I, Watanabe N, Nakata S, Komatsu R, Motoda S, Fujita Y, et al. Factors associated with treatment satisfaction in patients with type 2 diabetes mellitus using oral glucose-lowering agents: a cross-sectional study in urban districts in Japan. Endocr J. 2018;65(10):1001-9.

23. Biderman A, Noff E, Harris SB, Friedman N, Levy A. Treatment satisfaction of diabetic patients: what are the contributing factors? Fam Pract. 2009;26(2): 102-8.

24. Davies M, Speight J. Patient-reported outcomes in trials of incretin-based therapies in patients with type 2 diabetes mellitus. Diabetes Obes Metab. 2012;14(10):882-92.

25. Speight J, Reaney MD, Barnard KD. Not all roads lead to Rome-a review of quality of life measurement in adults with diabetes. Diabet Med. 2009;26(4):315-27.

26. Nicolucci A, Cucinotta D, Squatrito S, Lapolla A, Musacchio N, Leotta S, et al. Clinical and socioeconomic correlates of quality of life and treatment satisfaction in patients with type 2 diabetes. Nutr Metab Cardiovasc Dis. 2009;19(1):45-53.

27. Bener A, Al-Hamaq AO, Yousafzai MT, Abdul-Ghani M. Relationship between patient satisfactions with diabetes care and treatment. Niger J Clin Pract. 2014;17(2):218-25.

28. Narayan KM, Gregg EW, Fagot-Campagna A, Gary TL, Saaddine JB, Parker C, et al. Relationship between quality of diabetes care and patient satisfaction. J Natl Med Assoc. 2003;95(1):64-70.

29. Boels AM, Vos RC, Hermans TGT, Zuithoff NPA, Muller N, Khunti K, et al. What determines treatment satisfaction of patients with type 2 diabetes on insulin therapy? An observational study in eight European countries. BMJ Open. 2017;7(7):e016180.

30. Redekop WK, Koopmanschap MA, Stolk RP, Rutten GE, Wolffenbuttel BH, Niessen LW. Health-related quality of life and treatment satisfaction in Dutch 
patients with type 2 diabetes. Diabetes Care. 2002;25(3):458-63.

31. Ozder A, Sekeroglu M, Eker HH. Quality of life and satisfaction with treatment in subjects with type 2 diabetes: results from primary health care in Turkey. Int J Clin Exp Med. 2014;7(12):5715-22.

32. Ministry of Health, Labour and Welfare. The National Health and Nutrition Survey in Japan 2017. Tokyo, Japan: Ministry of Health, Labour and Welfare; 2017. Accessed Dec 2018. https://www. mhlw.go.jp/content/000681194.pdf.
33. Benjamini Y, Hochberg Y. Controlling the false discovery rate: a practical and powerful approach to multiple testing. J R Stat Soc Ser B. 1995;57: 289-300.

34. Ministry of Health, Labour and Welfare. Ethical guidelines for medical and health research involving human subjects. Tokyo, Japan: Ministry of Health, Labour and Welfare; 2015. Accessed Mar 2015. https://www.mhlw.go.jp/file/06-Seisakujouhou10600000-Daijinkanboukouseikagakuka/00000 80278.pdf. 